\title{
Study on College Attendance Platform Based on Bluetooth Technology and Android Platform
}

\author{
Jie Xiong \\ Huanggang Normal University, 438000 China \\ xiongjie@163.com
}

\begin{abstract}
To improve the efficiency of classroom attendance check, a college attendance platform based on Bluetooth technology and Android platform is put forward to quickly check the attendance at classroom by scanning the Bluetooth address of students' mobile phones. The teacher may check the attendance manually and remind each absent student by sending an SMS message, so as to improve the efficiency of attendance check. The SSH architecture featured by satisfying security is used in the system to realize the login from the Web end. This paper analyzes, studies and realizes the college roll call and attendance platform, and presents an empirical test of the classroom roll call system, which verifies the practical value of the platform in organizing the classroom teaching activities.
\end{abstract}

Key words: attendance management, Android, Bluetooth, SSH architecture.

\section{INTRODUCTION}

Classroom attendance plays an essential role in the teaching management of teachers, so it has been included in the routine teaching evaluation of teachers in many schools. Along with the rapid development of information technology, all kinds of mobile devices (smart phones, iPad, etc.) have been available to more people, bringing mobile education to public attention. Featured by flexibility, efficiency and interactivity, mobile education can help stimulate students' interest in learning and give full play to their subjectivity in learning [1]. Under this circumstance, educational APPs become popular among some teachers and students, but the theory for this practice has not been developed yet, so its application in education and teaching should be further explored.

A college attendance platform based on Android technology integrates electronic information technology, digitalization technology and network communication technology in the field of attendance management. Reference [2] proposed an attendance system based on Wi-Fi and Android to not only realize attendance check, but also achieve the communication between employees at lower cost. Reference [3] introduced an attendance system based on Android and GPS function, which utilized the GPS function for sign-in to effectively improve the efficiency of attendance check and reduce fake sign-ins. References [4] and [5] put forward the design and realization of the attendance system based on Android and Bluetooth to realize the function of attendance management through Android client, so as to facilitate the attendance management of teachers. This paper introduces the design and realization of attendance platform based on Android and Bluetooth and performs the modular analysis and description of system platform. 


\section{ANALYSIS OF PLATFORM FUNCTIONS}

\section{Overall Architecture Chart}

College attendance platform is divided into Teacher APP and Student APP. Teacher APP consists of such modules as Login, Course, Student, Attendance and Data Changes, while Student APP contains two modules, i.e. Login and Changes, as shown in Fig. 1.

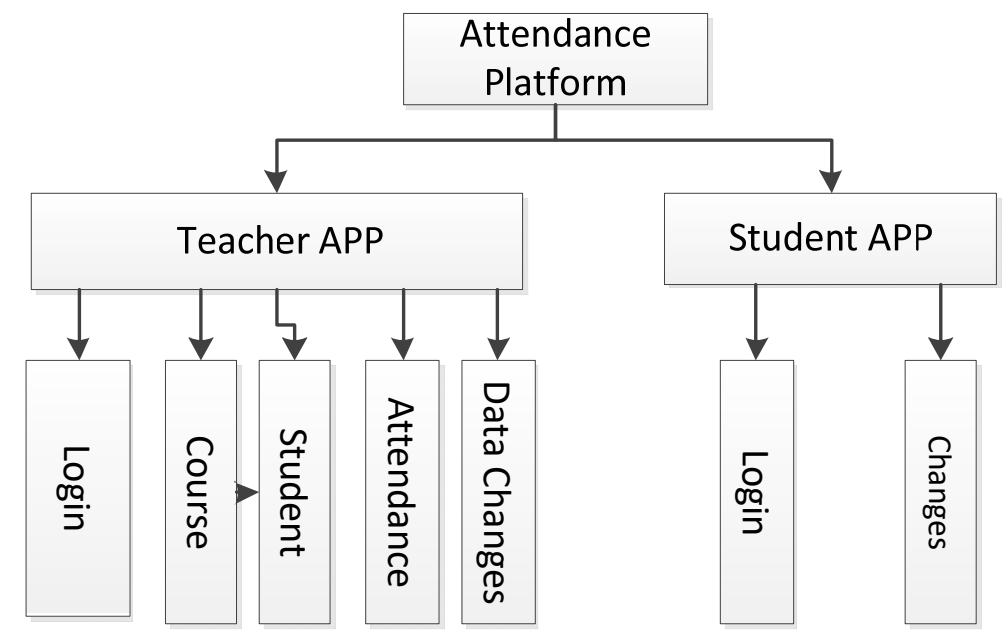

FIG. 1. Overall architecture chart

1) Login. This module contains username, password and security email. A user can log on by inputting username and password. If forgetting the password, the user may reset the password through the security email.

2) Student. This module allows managing student information, including Add, Change, Search and Delete student information. Student information includes student name, student ID, Bluetooth MAC address, class, major, school, and mobile phone number, etc. Among them, Bluetooth MAC address is the most important information. Student information can be imported in batches in the form of Excel datasheet, or manually added piece by piece.

3) Course. This module is used to manage course information, including Add, Change, Search and Delete course information. Course information includes course time, course teacher and registered students, etc. After the initial login through APP, course information is synchronized to local SQLite database, or user may update the course information of APP manually.

4) Attendance. This module is the core of this system. Each stream of attendance contains student name, student ID, and check time. These streams of attendance are stored in user's mobile phone. After roll call ends, teacher may forward the streams to the database at the back end of the system. MySQL is used by the database at the back end of the system. After being received, the streams of attendance are sorted and made into attendance list through trigger. An attendance list contains such information as student name, student ID, course name, course teacher, check time, check result (absence, lateness, attendance). At the end of each semester, teacher may generate an attendance record on the basis of attendance lists to contain student name, student ID, total attendance count, total absence count, and total lateness count, and takes the record as the basis for grading.

5) Data Changes. This module can forward attendance data to back-end database and gather information from back-end database. After the initial login through APP, course information and student information must be obtained from the back end and saved to local SQLite database. After each attendance check ends, APP should forward this stream of attendance to back-end database. 


\section{REALIZATION OF PLATFORM FUNCTIONS}

\section{Development Tools}

(1) Android. As a Linux-based operating system with free and open source code, Android is mainly used in mobile devices, e.g. smart phones and tablets. It was led and developed by Google and Open Handset Alliance.

(2) SQLite. Being contained in a relatively small C library, SQLite is a lightweight, ACID-compliant and relational database management system. As it occupies very few resources, SQLite may need only hundreds of kilobytes in a memory of embedded device. It can support Windows/Linux/Unix and other mainstream operating systems, and combine with such program design languages as C\#, PHP, and Java. SQLite also allows ODBC interface.

(3) Bluetooth. As a wireless technology standard, Bluetooth can exchange data over short distances (using shortwavelength UHF radio waves in the ISM band from 2.4 to $2.485 \mathrm{GHz}$ ) from fixed and mobile devices and building personal area networks (PANs).

(4) Tomcat. It is an open source and free Web application server and is often used when there are not many medium and small systems or concurrent users.

(5) SSH architecture. In the design of server end, lightweight SSH (i.e. Struts, Spring, Hibernate) architecture. In the SSH architecture, structs is responsible for MVC control separation, so it is the basic control architecture of SSH. Hibernate mainly supports the data persistence tier, while Spring is employed to manage Structs and Hibernate.

\section{Communication Mechanism}

Android client communicates through HTTP protocol. By calling the network interface provided by server end through URL address, Android client can communicate with the server, while the server uses Servlet for response to the request from the Android client and returns the data to the client. The specific steps are as follows:

1) User selects the Search module on the interface, so Internet initiates Activity;

2) The URL link in Activity is called, and the request is transmitted to the server through HTTP;

3) The server receives the request, calls the relevant method in Action, returns the corresponding Xml file, and resolves it;

4) The resolved content is displayed on the interface through Android space. Some key codes are as follows:

public void GetResult(requeset,response)

throws IOException \{

response.setContentType("text/xml”);

PringtWriter out=response.getWriter();

UpdataDao dao=UpdateDaoImpl ();

List list $=$ dao.getMenuList();

Out.println("<?xml version= "1.0" encoding= "UTF-8"?>")

\section{Database Design}

The entity objects included in the database of this platform include Login Information, Student Information, Course Information and Attendance Data, which are related to each other according to some rules. Their E-R diagram is as shown in Fig. 2. 


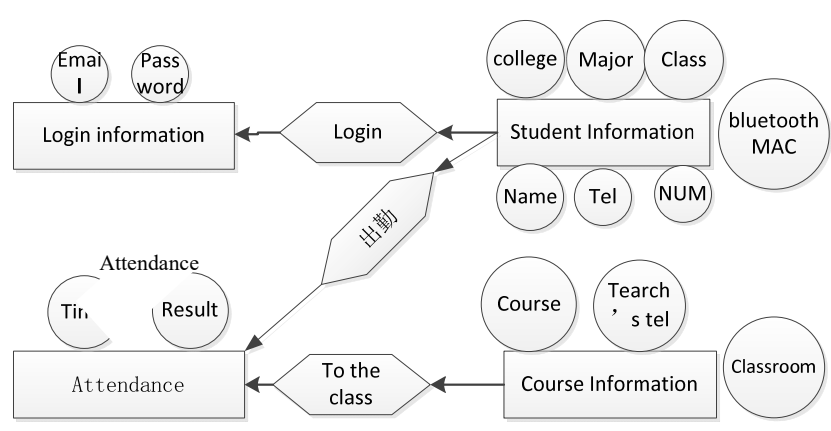

FIG. 2. Database relationship diagram

\section{ANALYSIS OF MODULAR FUNCTIONS}

\section{Add Student Information}

The management of student information includes Add, Change, Search and Delete student information. Add is divided into Batch Add and Add One by One. When the system is used for the first time, student information should be imported in batches. Management personnel can fill student information into Excel datasheets according to certain rules and call the Jxl.jar package to realize the importation in batches. The key codes are as follows:

StuDAO stuDAO=new StuDAO (Importsty.this)

Stu_Info stu_Info=new Stu_Info ();

/ / Read Excel Content

AddExcelToStu(stuDAO,stu_Info);

stuDAO.close(); / / Close Database Link

\section{Roll Call}

To realize the function of attendance check, the Bluetooth MAC address of every student's mobile phone should be saved in the database. When it is necessary to perform a roll call, students should turn on the Bluetooth feature of their mobile phones, and teacher can log on this system to perform the roll call function. The system gathers the MAC addresses of all students registered for the course, automatically scans the Bluetooth devices nearby, reads their MAC addresses, and compares them with MAC addresses in the database. When there is a match, the corresponding student's attendance is recorded. As a limited number of Bluetooth devices can be scanned each time, scanning should be repeated for several times to complete the attendance. After scanning is completed, the device will generate a list of absent students, and teacher may check the attendance of students manually as the case may be.

\section{Reminder by SMS Message}

When any student is absent, teacher may use the feature of Reminder by SMS message in the APP to send an SMS message to the student as a reminder. This system employs the Android message manager (SMS Manager) for sending SMS messages as follows: sendTextMessage (stu_phone,null, txtcontent,null,null), in which stu_phone is the student's mobile phone number, and txtcontent is the content of message.

\section{EMPIRICAL TEST OF CLASSROOM ROLL CALL SYSTEM}

The classroom roll call system was used in an empirical test by taking 29 students from 2016 Undergraduate Class in the Institute of Telecommunications of a provincial-level university, as shown in Fig. 3. 


\begin{tabular}{|lll|}
\hline 2016 -telecom-1601 & here $>$ \\
\hline 2016201001 & LIly & late $>$ \\
\hline 2016201002 & June & late $>$ \\
\hline 2016201003 & Lena & leave $>$ \\
\hline 2016201004 & Ally & here $>$ \\
\hline 2016201005 & Lisa & here $>$ \\
\hline 2016201006 & Jill & here $>$ \\
\hline 2016201007 & David & here $>$ \\
\hline 2016201008 & Catherine & here $>$ \\
\hline 2016201009 & Dinae & here $>$ \\
\hline 20162010010 Sharon & here > \\
\hline 20162010010 Lynn & here \\
\hline 2016an10011 Iet & Aa11 the ro11 \\
\hline
\end{tabular}

FIG. 3. Empirical Test

\section{CONCLUSION}

In general, it is simple to develop the college attendance platform based on Bluetooth technology and Android, and convenient to operate the system. Hence, the system features high degree of integration, easy scalability and clear layering. As proved in the empirical test, classroom roll call system has some value for application, and can be an effective tool for organizing the classroom teaching activities. Moreover, it plays an active role in the improvement of teaching efficiency, facilitates and arouses the students' curiosity and desire for knowledge to some extent. Hence, it is of certain referential value to the reform of classroom attendance system.

\section{ACKNOWLEDGEMENTS}

This paper is supported by the fund of Research project of Hubei Provincial Education Department (No. B2017196) and Key support project of Huanggang Nornal University in 2018 (No.2018-3).

\section{REFERENCES}

1. Zhang Hong. Study on the demand for mobile education based on mobile phone for primary schools in Beijing and its development [J]. E-education Research, 2011(7): 99-106.

2. Lan Lina. Development of Attendance and Communication System Based on web, Wi-Fi and Android [D]. Shijiazhuang: Hebei University of Science and Technology, 2013.

3. Bai Xiaoxuan. $[\mathrm{J}]$. The design and implementation of students sign system based on Android platform [J]. Software Engineer, 2015, 18 (7): 7-8.

4. Zhang Rui, Yu Xiaorong. Design and realization of intelligent mobile attendance system based on Android [J]. Information Technology, 2015, 5(4): 185-187.

5. Han Wenzhi, Luo Wenliang. Development method and application research of mobile APP based on Android platform [J]. Journal of Sichuan University of Science \& Engineering: Natural Science Edition, 2015, 28 (3): $22-24$. 